\title{
On the Delay Interval in Which the Control Delay Systems are Stabilizable
}

\author{
Jiang Wei $\mathbb{C}$ \\ School of Mathematical Science, Anhui University, Hefei, Anhui 230039, China \\ Correspondence should be addressed to Jiang Wei; jiangwei@ahu.edu.cn
}

Received 26 September 2019; Revised 10 December 2019; Accepted 21 December 2019; Published 10 March 2020

Guest Editor: Jorge-Antonio Lopez-Renteria

Copyright ( 92020 Jiang Wei. This is an open access article distributed under the Creative Commons Attribution License, which permits unrestricted use, distribution, and reproduction in any medium, provided the original work is properly cited.

In this paper, we firstly derive some criteria for considered control delay systems to be stabilizable and then get the interval of the control delay, in which the stabilizability of the systems can be kept.

\section{Introduction}

Recently, the phenomenon of time delay has aroused many people's attention [1-22]. In some practical systems, such as economic systems, biological systems, and space-light industry systems, due to the transmission of the signal or the mechanical transmission, the control variables must have time delay. From reference $[1,2]$, we can see that time delay usually destroys the stability. So, in the study of stabilizability of the control system, we must consider control delay seriously.

References [1-4] give the basic theory of functional differential equations that give us a lot of useful method and tools to discuss time delay systems. In [7], Jean-Pierre Richard gives an overview of some recent advances and open problems on time delay systems.

As we know, we can use the controllability to determine the stabilizability. Some scholars [2-4] and [8-11, 15, 22] have given many results on the controllability of the systems with delay.

System stability theory is the foundation of stabilizability of the control system. In papers [5, 6, 15-20, 22], the authors have given some excellent results for the stability of delay systems.

In this paper, we will point out that we can use the controllability of the systems with control delay to determine the stabilizability of such systems and give the method to do so. Therefore, we can use the results of references $[2,4]$ for the study of stabilizability of systems with control delay.
In reference $[5,6,15]$, John Chiasson, Jie Chen, and Jiang Wei give some results for computing the interval of delay values for stability of delay systems. These results will be useful in the study of the range of the control delay in which stabilizability of the systems can be kept.

The systems we consider in this paper will be the control systems with control delay:

$$
\begin{cases}\dot{x}(t)=A x(t)+B u(t-h), & t \geq t_{0}, \\ x\left(t_{0}\right)=x_{0}, & \\ u(t)=\varphi(t), & t_{0}-h \leq t \leq t_{0},\end{cases}
$$

where $x(t) \in R^{n}$ is a state vector; $u(t) \in R^{m}$ is a control vector; $u(\cdot)$ an admissible control, that is, it is contained in the square integrable functions $L^{2}$ on every finite interval; A $\in R^{n \times n}$ and $\mathrm{B} \in R^{n \times m}$ are constant matrices; $h>0$ is time delay; and $\varphi(t)$ is the initial control function.

In this paper, we will consider the stabilizability for the control systems with control delay (1). Firstly, we derive some criterions to criticize the stabilization for the control delay systems and then get the interval of the control delay, in which the stabilizability of the systems can be kept.

\section{Preliminaries}

For the control systems with control delay (1), we give some preliminaries involved in this paper. 
Definition 1. System (1) is said to be controllable at $t_{1} \geq t_{0}$ if one can reach any state at $t_{1}$ from any admissible initial state $x_{0}$ and initial control $\varphi(t)$.

Lemma 1. System (1) is controllable if and only if

$$
\operatorname{Rank}\left[B, A B, \ldots, A^{n-1} B\right]=n .
$$

The proof of this Lemma can be easily gotten from Theorem 1 in [4], but we must assume $n_{1}=n$ and $n_{2}=0$ there.

If there exist a matrix $K \in R^{n \times m}$, such that

$$
u(t)=K x(t),
$$

system (1) will become

$$
\dot{x}(t)=A x(t)+B K x(t-h) .
$$

Definition 2. The differential systems with delay (4) are called asymptotic stable, if there exist scalars $\alpha<0, \beta>0$ such that, for $t>t_{0}, x(t)$ satisfy

$$
|x(t)| \leq \beta e^{\alpha t}|x(0)|, \quad t \geq t_{0} .
$$

Definition 3. The control systems with control delay (1) are said to be stabilizable, if there is a matrix $K$ such that system (4) is asymptotically stable. The matrix $K$ is said to be a feedback stabilization matrix.

For an entire function $G(s)$, we define set

$$
J(G) \triangleq\{s \in C \mid G(s)=0\},
$$

where $C$ is the complex plane.

For system (4), we define the entire function:

$$
p(s ; A, B K ; h) \triangleq \operatorname{det}\left(s I-A-B K e^{-h s}\right) .
$$

From [1], we have the following.

Lemma 2. System (4) is asymptotically stable if and only if

$$
J(p(s ; A, B K ; h)) \subseteq C^{-},
$$

where $C^{-}$is the left complex plane.

If system (1) is controllable, that is, (2) is true, matrices $A$ and $B$ are equivalent to their controllable normalized forms. In the following, if (1) are controllable, we might as well let $A$ and $B$ be normalized forms:

$$
\begin{aligned}
A & =\left(\begin{array}{cccc}
A_{1} & A_{12} & \cdots & A_{1 l} \\
0 & A_{2} & \cdots & A_{2 l} \\
\cdots & \cdots & \cdots & \cdots \\
0 & 0 & \cdots & A_{l}
\end{array}\right)_{n \times n}, \\
B & =\left(\begin{array}{ccccc}
b_{1} & 0 & \cdots & 0 & * \\
0 & b_{2} & \cdots & 0 & * \\
\cdots & \cdots & \cdots & \cdots & \cdots \\
0 & 0 & \cdots & b_{l} & *
\end{array}\right)_{n \times m},
\end{aligned}
$$

where $A_{i}, b_{i}(i=1,2, \ldots, l)$ are the normalized forms as

$$
\begin{aligned}
A_{i} & =\left(\begin{array}{cccccc}
0 & 1 & 0 & \cdots & 0 & 0 \\
0 & 0 & 1 & \cdots & 0 & 0 \\
\cdots & \cdots & \cdots & \cdots & \cdots & \cdots \\
0 & 0 & 0 & \cdots & 0 & 1 \\
-a_{i, 0} & -a_{i, 1} & -a_{i, 2} & \cdots & -a_{i, n_{i}-2} & -a_{i, n_{i}-1}
\end{array}\right), \\
b_{i} & =\left(\begin{array}{c}
0 \\
0 \\
\vdots \\
0 \\
1
\end{array}\right) .
\end{aligned}
$$

Let

$$
K=\left(\begin{array}{c}
\widehat{K} \\
0
\end{array}\right)
$$

where

$$
\begin{aligned}
\widehat{K} & =\left(\begin{array}{ccccc}
\bar{k}_{1}^{T} & 0 & \cdots & 0 & 0 \\
0 & \bar{k}_{2}^{T} & \cdots & 0 & 0 \\
\cdots & \cdots & \cdots & \cdots & \cdots \\
0 & 0 & \cdots & 0 & \bar{k}_{l}^{T}
\end{array}\right), \\
\bar{k}_{i} & =\left(k_{1}, k_{2}, \cdots, k_{n_{i}}\right) .
\end{aligned}
$$

Then,

$$
p(s ; A, B K ; h)=\prod_{i=1}^{l} p\left(s ; A_{i}, b_{i} k_{i}^{T} ; h\right) .
$$

From (13) and Lemma 2, we have the following.

Lemma 3. If the control systems with control delay (1) are controllable, they are stabilizable if and only if

$$
J\left(p\left(s ; A_{i}, b_{i} k_{i}^{T} ; h\right)\right) \subseteq C^{-},
$$

for every $i=1,2, \ldots, l$, is true.

$$
\begin{aligned}
& \text { Let } d=e^{-s h} \text { and } \\
& \alpha(s, d)=s^{n}+a_{n-1}(d) s^{n-1}+\cdots+a_{0}(d),
\end{aligned}
$$

where $a_{i}(d)(i=1,2, \cdots, n-1)$ are the polynomials of $d$.

Definition 4. Let $\alpha(s, d)$ be a two-variable polynomial of form (15) and $r=\operatorname{deg}_{d}\{\alpha(s, d)\}$, and we call the two-variable polynomial,

$$
\begin{aligned}
\widetilde{\alpha}(s, d) & \triangleq d^{r} \alpha(-s, 1 / d) \\
& =(-1)^{n} d^{r} s^{n}+d^{r} a_{n-1}(1 / d)(-s)^{n-1}+\cdots+d^{r} a_{0}(1 / d),
\end{aligned}
$$

the auxiliary polynomial of $\alpha(s, d)$.

Suppose $\left\{\left(s_{i}, d_{i}\right), i=1,2, \ldots, k\right\}$ be the common zeros of $\{\alpha(s, d), \widetilde{\alpha}(s, d)\}$ for which $\operatorname{Re}\left(s_{i}\right)=0, s_{i} \neq 0$ and $\left|d_{i}\right|=1$, 
$d_{i} \neq 1$. For each such pair $\left(s_{i}, d_{i}\right)$, let $h_{i}=\min _{h>0}\{h \in R \mid$ $\left.d_{i}=e^{-\mathrm{hs}_{\mathrm{i}}}\right\}$. Since $s_{i} \neq 0$ and $\left|d_{i}\right|=1$, such an $h_{i}$ exists. Define

$$
h^{*}=\min _{1 \leq i \leq k}\left\{h_{i}\right\}>0 \text {. }
$$

From [5], we have the following.

\section{Lemma 4}

$$
\alpha\left(s, e^{-h s}\right) \neq 0, \quad R(s) \geq 0, \quad 0 \leq h<h^{*},
$$

if and only if

$$
\begin{gathered}
\alpha(s, 1) \neq 0, \\
R(s) \geq 0 .
\end{gathered}
$$

Furthermore, there exists an $s^{*}=j \omega^{*}$ for which $\alpha\left(s^{*}, e^{-h^{*} s^{*}}\right)=0$.

\section{Main Results}

In this section, we will give the main results of this paper. Firstly, we give a general theorem, then for $n=2$, discuss in two cases, and give some results.

Theorem 1. For system (1), if they are controllable, there exists a real number $h^{*}>0$ such that they are stabilizable with delay $h \in\left[0, h^{*}\right)$.

Proof. From Lemma 1, system (1) is controllable if and only if (2) is true. It is obvious that if (2) is true, there exists a matrix $K$ such that

$$
J(\operatorname{det}(s I-A-B K)) \subseteq C^{-} .
$$

Let $\alpha(s, d)=\operatorname{det}(s I-A-B K d)$ and take $h^{*}$ as that in (17); then, from Lemma 4, we have that Theorem 1 is true.

From Lemma 3, to discuss the stabilizability of system (1) in detail, if they are controllable, we might as well let

$$
\begin{aligned}
A & =\left(\begin{array}{cccccc}
0 & 1 & 0 & \cdots & 0 & 0 \\
0 & 0 & 1 & \cdots & 0 & 0 \\
\cdots & \cdots & \cdots & \cdots & \cdots & \cdots \\
0 & 0 & 0 & \cdots & 0 & 1 \\
-a_{0} & -a_{1} & -a_{2} & \cdots & -a_{n-2} & -a_{n-1}
\end{array}\right), \\
B & =\left(\begin{array}{c}
0 \\
0 \\
\vdots \\
0 \\
1
\end{array}\right)
\end{aligned}
$$

Take $K=\left\{-k_{0},-k_{1}, \ldots,-k_{n-1}\right\}^{T}$, where $k_{0}, k_{1}, \ldots, k_{n-1}$ are undetermined real numbers, and we have

$$
\begin{aligned}
p\left(s ; A, B K^{T} ; h\right)= & \operatorname{det}\left(s I-A-B K^{T} e^{-s h}\right) \\
= & s^{n}+a_{n-1} s^{n-1}+\cdots+a_{0}+e^{-s h} \\
& \cdot\left(k_{n-1} s^{n-1}+\cdots+k_{0}\right) .
\end{aligned}
$$

If there exist some eigenvalues of $A$ with the negative real part, let $\lambda_{1}, \lambda_{2}, \ldots, \lambda_{p}$ be all the eigenvalues of $A$ with $\operatorname{Re}\left(\lambda_{i}\right)<0,(i=1,2, \ldots, p)$, then there exist real numbers $a_{0}^{\prime}, a_{1}^{\prime}, \ldots, a_{n-p-1}^{\prime}$ such that

$$
\begin{aligned}
p(s ; A)= & s^{n}+a_{n-1} s^{n-1}+\cdots+a_{0} \\
= & \left(s-\lambda_{1}\right)\left(s-\lambda_{2}\right) \cdots\left(s-\lambda_{p}\right) \\
& \cdot\left(s^{n-p}+a_{n-p-1}^{\prime} s^{n-p-1}+\cdots+a_{0}^{\prime}\right) .
\end{aligned}
$$

Here, $s^{n-p}+a_{n-p-1}^{\prime} s^{n-p-1}+\cdots+a_{0}^{\prime}=0$ and there exists only nonnegative real part roots.

Obviously, for any leading coefficient which is 1 and $n-$ $p$ order polynomial $g(s)$, we can set

$$
\begin{aligned}
s^{n} & +a_{n-1} s^{n-1}+\cdots+a_{0}+\left(k_{n-1} s^{n-1}+\cdots+k_{0}\right) \\
& =\left(s-\lambda_{1}\right)\left(s-\lambda_{2}\right) \cdots\left(s-\lambda_{p}\right) g(s),
\end{aligned}
$$

that is,

$$
\begin{aligned}
& \left(s-\lambda_{1}\right)\left(s-\lambda_{2}\right) \cdots\left(s-\lambda_{p}\right)\left(s^{n-p}+a_{n-p-1}^{\prime} s^{n-p-1}+\cdots+a_{0}^{\prime}\right) \\
& \quad+\left(k_{n-1} s^{n-1}+\cdots+k_{0}\right)=\left(s-\lambda_{1}\right)\left(s-\lambda_{2}\right) \cdots\left(s-\lambda_{p}\right) g(s) .
\end{aligned}
$$

Let

$$
g(s)=s^{n-p}+b_{n-p-1} s^{n-p-1}+\cdots+b_{0},
$$

there $b_{n-p-1}, \ldots, b_{0}$ are arbitrary constants. Then, we have

$$
\begin{aligned}
k_{n-1} & s^{n-1}+\cdots+k_{0} \\
& =\left(s-\lambda_{1}\right)\left(s-\lambda_{2}\right) \cdots\left(s-\lambda_{p}\right) g(s)-\left(s-\lambda_{1}\right)\left(s-\lambda_{2}\right) \cdots\left(s-\lambda_{p}\right)\left(s^{n-p}+a_{n-p-1}^{\prime} s^{n-p-1}+\cdots+a_{0}^{\prime}\right) \\
& =\left(s-\lambda_{1}\right)\left(s-\lambda_{2}\right) \cdots\left(s-\lambda_{p}\right)\left(s^{n-p}+b_{n-p-1} s^{n-p-1}+\cdots+b_{0}\right)-\left(s-\lambda_{1}\right)\left(s-\lambda_{2}\right) \cdots\left(s-\lambda_{p}\right)\left(s^{n-p}+a_{n-p-1}^{\prime} s^{n-p-1}+\cdots+a_{0}^{\prime}\right) \\
& =\left(s-\lambda_{1}\right)\left(s-\lambda_{2}\right) \cdots\left(s-\lambda_{p}\right)\left(\left(b_{n-p-1}-a_{n-p-1}^{\prime}\right) s^{n-p-1}+\cdots+\left(b_{0}-a_{0}^{\prime}\right)\right) .
\end{aligned}
$$


Let $\quad k_{0}^{\prime}=b_{0}-a_{0}^{\prime}, k_{1}^{\prime}=b_{1}-a_{1}^{\prime}, \ldots, k_{n-p-1}^{\prime}=b_{n-p-1}-$ $a_{n-p-1}^{\prime}$, then

$$
\begin{aligned}
k_{n-1} s^{n-1}+\cdots+k_{0}= & \left(s-\lambda_{1}\right)\left(s-\lambda_{2}\right) \cdots\left(s-\lambda_{p}\right) \\
& \left(k_{n-p-1}^{\prime} s^{n-p-1}+\cdots+k_{0}^{\prime}\right) .
\end{aligned}
$$

From (28), we can determine real numbers $k_{0}, k_{1}, \ldots, k_{n-1}$.

Take (28) into (22), we have

$$
\begin{aligned}
p(s ; & \left.A, B K^{T} ; h\right)=\operatorname{det}\left(s I-A-B K^{T} e^{-s h}\right) \\
= & s^{n}+a_{n-1} s^{n-1}+\cdots+a_{0}+e^{-s h}\left(k_{n-1} s^{n-1}+\cdots+k_{0}\right) \\
= & \left(s-\lambda_{1}\right)\left(s-\lambda_{2}\right) \cdots\left(s-\lambda_{p}\right)\left(s^{n-p}+a_{n-p-1}^{\prime} s^{n-p-1}+\cdots+a_{0}^{\prime}\right) \\
& +e^{-s h}\left(s-\lambda_{1}\right)\left(s-\lambda_{2}\right) \cdots\left(s-\lambda_{p}\right)\left(k_{n-p-1}^{\prime} s^{n-p-1}+\cdots+k_{0}^{\prime}\right) \\
= & \left(s-\lambda_{1}\right)\left(s-\lambda_{2}\right) \cdots\left(s-\lambda_{p}\right)\left\{s^{n-p}+a_{n-p-1}^{\prime} s^{n-p-1}+\cdots+a_{0}^{\prime}\right. \\
& \left.+e^{-s h}\left(k_{n-p-1}^{\prime} s^{n-p-1}+\cdots+k_{0}^{\prime}\right)\right\} .
\end{aligned}
$$

Let

$$
\begin{aligned}
p\left(s ; A^{\prime}, B^{\prime} K^{\prime T} ; h\right)= & s^{n-p}+a_{n-p-1}^{\prime} s^{n-p-1}+\cdots+a_{0}^{\prime} \\
& +e^{-s h}\left(k_{n-p-1}^{\prime} s^{n-p-1}+\cdots+k_{0}^{\prime}\right) .
\end{aligned}
$$

We can see that, if we want

$$
J\left(p\left(s ; A, B K^{T} ; h\right)\right) \subseteq C^{-},
$$

it is enough to make

$$
J\left(p\left(s ; A^{\prime}, B^{\prime} K^{\prime T} ; h\right)\right) \subseteq C^{-} .
$$

So, in the following discussion, we might as well assume that $A$ have only nonnegative real part eigenvalues, that is,

$$
p(s ; A)=s^{n}+a_{n-1} s^{n-1}+\cdots+a_{0}=0,
$$

there exists only nonnegative real part roots.

Now we discuss the stabilizability of system (1) with $n=2$. We discuss that in two cases.

Case I. $n=2$ and $P(s, A)$ and there exists only positive real part zeros.

If the quadratic polynomial equation

$$
p(s ; A)=s^{2}+a_{1} s+a_{0}=0,
$$

there exists only positive real part roots, and we can rewrite $P(s, A)$ as

$$
p(s ; A)=(s-a)^{2}+b=0, \quad a>0, b \geq 0 .
$$

Theorem 2. For system (1), when $n=2$ and $p(s ; A)=$ $(s-a)^{2}+b=0, a>0, b \geq 0$, if they are controllable, then they are stabilizable with delay $h \in\left[0,\left(\pi / 3\left(\sqrt{4 a^{2}+b}+\sqrt{3} a\right)\right)\right)$.

Proof. For $p(s ; A)=(s-a)^{2}+b=0, a>0, b \geq 0$, we have that

$$
p(-s ; A)=(s+a)^{2}+b=0
$$

and there exists only negative real part roots.

Let

$$
(s-a)^{2}+b+\left(k_{1} s+k_{0}\right)=(s+a)^{2}+b,
$$

then $k_{1}=4 a$ and $k_{0}=0$.

$$
\alpha(s, d)=(s-a)^{2}+b+d(4 a s)=0,
$$

$$
\widetilde{\alpha}(s, d)=d \alpha(-s, 1 / d)=d\left((s+a)^{2}+b\right)-(4 a s)=0 .
$$

From (38), we have

$$
d=-\frac{(s-a)^{2}+b}{4 a s} .
$$

Taking it into (39), we have

$$
s^{4}+\left(14 a^{2}+2 b\right) s^{2}+\left(a^{2}+b\right)^{2}=0 .
$$

The solutions of (41) are

$$
\begin{aligned}
& s_{1}=\sqrt{\left(7 a^{2}+b\right)+2 \sqrt{3\left(4 a^{2}+b\right)} a j=\left(\sqrt{4 a^{2}+b}+\sqrt{3} a\right) j}, \\
& s_{2}=\sqrt{\left(7 a^{2}+b\right)-2 \sqrt{3\left(4 a^{2}+b\right)} a j=\left(\sqrt{4 a^{2}+b}-\sqrt{3} a\right) j}, \\
& s_{3}=-\sqrt{\left(7 a^{2}+b\right)+2 \sqrt{3\left(4 a^{2}+b\right)} a j=-\left(\sqrt{4 a^{2}+b}+\sqrt{3} a\right) j}, \\
& s_{4}=-\sqrt{\left(7 a^{2}+b\right)-2 \sqrt{3\left(4 a^{2}+b\right)} a j=-\left(\sqrt{4 a^{2}+b}-\sqrt{3} a\right) j} .
\end{aligned}
$$

Here, $j^{2}=-1$. Taking $s_{1}, s_{2}, s_{3}$, and $s_{4}$ into (40), we have

$$
\begin{aligned}
& d_{1}=-\frac{\left(\left(\sqrt{4 a^{2}+b}+\sqrt{3} a\right) j-a\right)^{2}+b}{4 a\left(\sqrt{4 a^{2}+b}+\sqrt{3} a\right) j}=\frac{1}{2}-\frac{\sqrt{3}}{2} j, \\
& d_{2}=-\frac{\left(\left(\sqrt{4 a^{2}+b}-\sqrt{3} a\right) j-a\right)^{2}+b}{4 a\left(\sqrt{4 a^{2}+b}-\sqrt{3} a\right) j}=\frac{1}{2}+\frac{\sqrt{3}}{2} j,
\end{aligned}
$$

$d_{3}=-\frac{\left(\left(\sqrt{4 a^{2}+b}+\sqrt{3} a\right) j+a\right)^{2}+b}{-4 a\left(\sqrt{4 a^{2}+b}+\sqrt{3} a\right) j}=\frac{1}{2}+\frac{\sqrt{3}}{2} j$

$d_{4}=-\frac{\left(\left(\sqrt{4 a^{2}+b}-\sqrt{3} a\right) j+a\right)^{2}+b}{-4 a\left(\sqrt{4 a^{2}+b}-\sqrt{3} a\right) j}=\frac{1}{2}-\frac{\sqrt{3}}{2} j$.

For

$$
\begin{aligned}
d_{1} & =e^{-h s_{1}} \Longleftrightarrow \frac{1}{2}-\frac{\sqrt{3}}{2} j=e^{-h\left(\sqrt{4 a^{2}+b}+\sqrt{3} a\right) j}, \\
& \Longleftrightarrow\left\{\begin{array}{l}
\cos \left[h\left(\sqrt{4 a^{2}+b}+\sqrt{3} a\right)\right]=\frac{1}{2}, \\
\sin \left[h\left(\sqrt{4 a^{2}+b}+\sqrt{3} a\right)\right]=\frac{\sqrt{3}}{2},
\end{array}\right. \\
& \Longleftrightarrow h\left(\sqrt{4 a^{2}+b}+\sqrt{3} a\right)=2 k \pi+\frac{\pi}{3}, \quad k=0,1,2, \ldots .
\end{aligned}
$$


Then, we have

$$
h_{1}=\min _{h>0}\left\{h \in R \mid d_{1}=e^{-h s_{1}}\right\}=\frac{\pi}{3\left(\sqrt{4 a^{2}+b}+\sqrt{3} a\right)} \text {. }
$$

Using the same method, we have

$$
\begin{aligned}
& h_{2}=\min _{h>0}\left\{h \in R \mid d_{2}=e^{-h s_{2}}\right\}=\frac{5 \pi}{3\left(\sqrt{4 a^{2}+b}-\sqrt{3} a\right)}, \\
& h_{3}=\min _{h>0}\left\{h \in R \mid d_{3}=e^{-h s_{3}}\right\}=\frac{\pi}{3\left(\sqrt{4 a^{2}+b}+\sqrt{3} a\right)}, \\
& h_{4}=\min _{h>0}\left\{h \in R \mid d_{3}=e^{-h s_{3}}\right\}=\frac{5 \pi}{3\left(\sqrt{4 a^{2}+b}-\sqrt{3} a\right)} .
\end{aligned}
$$

So,

$$
h^{*}=\min \left\{h_{1}, h_{2}, h_{3}, h_{4}\right\}=\frac{\pi}{3\left(\sqrt{4 a^{2}+b}+\sqrt{3} a\right)} .
$$

Then, we have that Theorem 2 is true.

Case II. $n=2$ and $P(s, A)$ has only pure imaginary zeros.

If the quadratic polynomial equation

$$
p(s ; A)=s^{2}+a_{1} s+a_{0}=0,
$$

there exists only pure imaginary roots, and we can rewrite $P(s, A)$ as

$$
p(s ; A)=s^{2}+b=0, \quad b>0 .
$$

Theorem 3. For system (1), when $n=2$ and $p(s ; A)=s^{2}+$ $b=0, b>0$, if they are controllable, then they are stabilizable with delay $h \in[0, \pi / 2(\sqrt{b}+\sqrt{2 b}))$.

Proof. Let

$$
s^{2}+b+\left(k_{1} s+k_{0}\right)=(s+\sqrt{b})^{2}=s^{2}+2 \sqrt{b} s+b,
$$

then $k_{1}=2 \sqrt{b}$ and $k_{0}=0$.

$$
\begin{aligned}
& \alpha(s, d)=s^{2}+b+d(2 \sqrt{b} s)=0, \\
& \widetilde{\alpha}(s, d)=d \alpha(-s, 1 / d)=d\left(s^{2}+b\right)-2 \sqrt{b} s=0 .
\end{aligned}
$$

From (51), we have

$$
d=-\frac{s^{2}+b}{2 \sqrt{b} s}
$$

Taking it into (52), we have

$$
\left(s^{2}+b\right)^{2}+4 b s^{2}=0 .
$$

The solutions of (54) are

$$
\begin{aligned}
& s_{1}=(\sqrt{b}+\sqrt{2 b}) j, \\
& s_{2}=-(\sqrt{2 b}-\sqrt{b}) j, \\
& s_{3}=-(\sqrt{b}+\sqrt{2 b}) j, \\
& s_{4}=(\sqrt{2 b}-\sqrt{b}) j .
\end{aligned}
$$

Taking them into (53), we have

$$
\begin{aligned}
& d_{1}=d_{2}=-j, \\
& d_{3}=d_{4}=j .
\end{aligned}
$$

For

$$
\begin{aligned}
d_{1}=e^{-h s_{1}} & \Longleftrightarrow-j=e^{-h(\sqrt{b}+\sqrt{2 b}) j}, \\
& \Longleftrightarrow\left\{\begin{array}{l}
\cos [h(\sqrt{b}+\sqrt{2 b})]=0, \\
\sin [h(\sqrt{b}+\sqrt{2 b})]=1, \\
\\
\Longleftrightarrow h(\sqrt{b}+\sqrt{2 b})=2 k \pi+\frac{\pi}{2}, \quad k=0,1,2, \ldots
\end{array}\right.
\end{aligned}
$$

Then, we have

$$
h_{1}=\min _{h>0}\left\{h \in R \mid d_{1}=e^{-h s_{1}}\right\}=\frac{\pi}{2(\sqrt{b}+\sqrt{2 b})} .
$$

Using the same method, we have

$$
\begin{aligned}
& h_{2}=\min _{h>0}\left\{h \in R \mid d_{2}=e^{-h s_{2}}\right\}=\frac{3 \pi}{2(\sqrt{2 b}-\sqrt{b})}, \\
& h_{3}=\min _{h>0}\left\{h \in R \mid d_{3}=e^{-h s_{3}}\right\}=\frac{3 \pi}{2(\sqrt{b}+\sqrt{2 b})}, \\
& h_{4}=\min _{h>0}\left\{h \in R \mid d_{3}=e^{-h s_{3}}\right\}=\frac{\pi}{2(\sqrt{2 b}-\sqrt{b})} .
\end{aligned}
$$

So,

$$
h^{*}=\min \left\{h_{1}, h_{2}, h_{3}, h_{4}\right\}=\frac{\pi}{2(\sqrt{b}+\sqrt{2 b})} .
$$

Then, we have Theorem 3 is true.

Now, we discuss the general case: for any positive integer $n$, the polynomial

$$
p(s ; A)=s^{n}+a_{n-1} s^{n-1}+\cdots+a_{1} s+a_{0}=0,
$$

and there exists only positive real part roots.

It is obvious that the polynomial equation

$$
\widehat{p}(s ; A)=s^{n}-a_{n-1} s^{n-1}+\cdots+(-1)^{n-1} a_{1} s+(-1)^{n} a_{0}=0,
$$

and there exists only negative real part roots.

Let

$$
\begin{aligned}
& s^{n}+a_{n-1} s^{n-1}+\cdots+a_{1} s+a_{0}+\left(k_{n-1} s^{n-1}+\cdots+k_{1} s+k_{0}\right) \\
& =s^{n}-a_{n-1} s^{n-1}+\cdots+(-1)^{n-1} a_{1} s+(-1)^{n} a_{0},
\end{aligned}
$$

then $k_{i}=\left((-1)^{n-i}-1\right) a_{i}(i=0,1, \ldots, n-1)$.

When $n$ is an even number, let $n=2 r$, and we have 


$$
\begin{aligned}
\alpha(s, d)= & s^{2 r}+a_{2 r-1} s^{2 r-1}+\cdots+a_{1} s+a_{0} \\
& -2 d\left(a_{2 r-1} s^{2 r-1}+a_{2 r-3} s^{2 r-3}+\cdots+a_{1} s\right)=0 \\
\widetilde{\alpha}(s, d)= & \left(s^{2 r}-a_{2 r-1} s^{2 r-1}+\cdots-a_{1} s+a_{0}\right) d \\
& +2\left(a_{2 r-1} s^{2 r-1}+a_{2 r-3} s^{2 r-3}+\cdots+a_{1} s\right)=0 .
\end{aligned}
$$

From (64), we have

$$
d=\frac{s^{2 r}+a_{2 r-1} s^{2 r-1}+\cdots+a_{1} s+a_{0}}{2\left(a_{2 r-1} s^{2 r-1}+a_{2 r-3} s^{2 r-3}+\cdots+a_{1} s\right)} .
$$

Taking it into (65), we obtain

$$
\begin{aligned}
& \left(s^{2 r}-a_{2 r-1} s^{2 r-1}+\cdots-a_{1} s+a_{0}\right) \\
& \left(s^{2 r}+a_{2 r-1} s^{2 r-1}+\cdots+a_{1} s+a_{0}\right) \\
& \quad+4\left(a_{2 r-1} s^{2 r-1}+a_{2 r-3} s^{2 r-3}+\cdots+a_{1} s\right)^{2}=0 .
\end{aligned}
$$

We can prove that all the roots of (67) are pure imaginary roots, let them be $s_{i}=j y_{i},(i=1,2, \ldots, 2 r)$; then (67) will become

$$
\begin{aligned}
& \left(\left(j y_{i}\right)^{2 r}-a_{2 r-1}\left(j y_{i}\right)^{2 r-1}+\cdots-a_{1}\left(j y_{i}\right)+a_{0}\right) \\
& \quad \times\left(\left(j y_{i}\right)^{2 r}+a_{2 r-1}\left(j y_{i}\right)^{2 r-1}+\cdots+a_{1}\left(j y_{i}\right)+a_{0}\right) \\
& \quad+4\left(a_{2 r-1}\left(j y_{i}\right)^{2 r-1}+a_{2 r-3}\left(j y_{i}\right)^{2 r-3}+\cdots+a_{1}\left(j y_{i}\right)\right)^{2}=0 .
\end{aligned}
$$

For

$$
\begin{aligned}
\left(\left(j y_{i}\right)^{2 r}-a_{2 r-1}\left(j y_{i}\right)^{2 r-1}+\cdots-a_{1}\left(j y_{i}\right)+a_{0}\right) \times\left(\left(j y_{i}\right)^{2 r}+a_{2 r-1}\left(j y_{i}\right)^{2 r-1}+\cdots+a_{1}\left(j y_{i}\right)+a_{0}\right) \\
=\left\{\left(\left(j y_{i}\right)^{2 r}+a_{2 r-2}\left(j y_{i}\right)^{2 r-2}+\cdots+a_{2}\left(j y_{i}\right)^{2}+a_{0}\right)-\left(a_{2 r-1}\left(j y_{i}\right)^{2 r-1}+a_{2 r-3}\left(j y_{i}\right)^{2 r-3}+\cdots+a_{1}\left(j y_{i}\right)\right)\right\} \\
\quad \times\left\{\left(\left(j y_{i}\right)^{2 r}+a_{2 r-2}\left(j y_{i}\right)^{2 r-2}+\cdots+a_{2}\left(j y_{i}\right)^{2}+a_{0}\right)+\left(a_{2 r-1}\left(j y_{i}\right)^{2 r-1}+a_{2 r-3}\left(j y_{i}\right)^{2 r-3}+\cdots+a_{1}\left(j y_{i}\right)\right)\right\} .
\end{aligned}
$$

We obtain

$$
\begin{aligned}
& \left(\left(j y_{i}\right)^{2 r}+a_{2 r-2}\left(j y_{i}\right)^{2 r-2}+\cdots+a_{2}\left(j y_{i}\right)^{2}+a_{0}\right)^{2} \\
& \quad-\left(a_{2 r-1}\left(j y_{i}\right)^{2 r-1}+a_{2 r-3}\left(j y_{i}\right)^{2 r-3}+\cdots+a_{1}\left(j y_{i}\right)\right)^{2} \\
& \quad+4\left(a_{2 r-1}\left(j y_{i}\right)^{2 r-1}+a_{2 r-3}\left(j y_{i}\right)^{2 r-3}+\cdots+a_{1}\left(j y_{i}\right)\right)^{2}=0
\end{aligned}
$$

then

$$
\begin{aligned}
& \left(\left(j y_{i}\right)^{2 r}+a_{2 r-2}\left(j y_{i}\right)^{2 r-2}+\cdots+a_{2}\left(j y_{i}\right)^{2}+a_{0}\right)^{2} \\
& =-3\left(a_{2 r-1}\left(j y_{i}\right)^{2 r-1}+a_{2 r-3}\left(j y_{i}\right)^{2 r-3}+\cdots+a_{1}\left(j y_{i}\right)\right)^{2} .
\end{aligned}
$$

So,

$$
\begin{aligned}
& \left(\left(j y_{i}\right)^{2 r}+a_{2 r-2}\left(j y_{i}\right)^{2 r-2}+\cdots+a_{2}\left(j y_{i}\right)^{2}+a_{0}\right) \\
& = \pm \sqrt{3} j\left(a_{2 r-1}\left(j y_{i}\right)^{2 r-1}+a_{2 r-3}\left(j y_{i}\right)^{2 r-3}+\cdots+a_{1}\left(j y_{i}\right)\right) .
\end{aligned}
$$

From (66), we have

$$
\begin{aligned}
d_{i}= & \frac{\left(j y_{i}\right)^{2 r}+a_{2 r-1}\left(j y_{i}\right)^{2 r-1}+\cdots+a_{1}\left(j y_{i}\right)+a_{0}}{2\left(a_{2 r-1}\left(j y_{i}\right)^{2 r-1}+a_{2 r-3}\left(j y_{i}\right)^{2 r-3}+\cdots+a_{1}\left(j y_{i}\right)\right)} \\
= & \frac{1}{2\left(a_{2 r-1}\left(j y_{i}\right)^{2 r-1}+a_{2 r-3}\left(j y_{i}\right)^{2 r-3}+\cdots+a_{1}\left(j y_{i}\right)\right)} \\
& \times\left[\left(a_{2 r-1}\left(j y_{i}\right)^{2 r-1}+a_{2 r-3}\left(j y_{i}\right)^{2 r-3}+\cdots+a_{1}\left(j y_{i}\right)\right)+\left(\left(j y_{i}\right)^{2 r}+a_{2 r-2}\left(j y_{i}\right)^{2 r-2}+\cdots+a_{2}\left(j y_{i}\right)^{2}+a_{0}\right)\right] \\
= & \frac{1}{2\left(a_{2 r-1}\left(j y_{i}\right)^{2 r-1}+a_{2 r-3}\left(j y_{i}\right)^{2 r-3}+\cdots+a_{1}\left(j y_{i}\right)\right)} \\
& \times\left[\left(a_{2 r-1}\left(j y_{i}\right)^{2 r-1}+a_{2 r-3}\left(j y_{i}\right)^{2 r-3}+\cdots+a_{1}\left(j y_{i}\right)\right) \pm \sqrt{3} j\left(a_{2 r-1}\left(j y_{i}\right)^{2 r-1}+a_{2 r-3}\left(j y_{i}\right)^{2 r-3}+\cdots+a_{1}\left(j y_{i}\right)\right)\right] \\
= & \frac{1}{2} \pm \frac{\sqrt{3}}{2} j,
\end{aligned}
$$


where $i=1,2, \ldots, n$.

In the same way, we can also prove that when $n$ is an odd number, we still have $d_{i}=1 / 2 \pm \sqrt{3} / 2 j,(i=1,2, \ldots, n)$.

From $d_{i}=e^{-h s_{i}}=e^{-\left(h y_{i}\right) j}$ and $h_{i}=\min _{h>0}\left\{h \in R \mid d_{i}=\right.$ $\left.e^{-\left(h y_{i}\right) j}\right\}$, we have $h_{i}\left|y_{j}\right|=\pi / 3$ or $h_{i}\left|y_{j}\right|=5 \pi / 3$.

Let $y^{*}=\max \left\{y_{1}, y_{2}, \ldots, y_{n}\right\}$. It is obvious that $y^{*}>0$. Then, we have

$$
h^{*}=\min \left\{h_{1}, h_{2}, \ldots, h_{n}\right\}=\frac{\pi}{3 y^{*}} .
$$

From the discussion above, we obtain the following.

Theorem 4. For system (1), if they are controllable and

$$
p(s ; A)=s^{n}+a_{n-1} s^{n-1}+\cdots+a_{1} s+a_{0}=0,
$$

there exists only positive real part roots, and they are stabilizable with control delay $h \in\left[0, \pi / 3 y^{*}\right)$.

Now, we give an example to illustrate the use of results.

\section{Example 1. Consider systems}

$$
\begin{cases}\dot{x}_{1}(t)=x_{2}(t), & t \geq 0, \\ \dot{x}_{2}(t)=-x_{1}(t)+2 x_{2}(t), & t \geq 0, \\ x_{1}(0)=1, & \\ x_{2}(0)=1, & \end{cases}
$$

where $x_{1}(t), x_{2}(t) \in R$.

The solutions of (76) are

$$
\left\{\begin{array}{l}
x_{1}(t)=e^{t}, t \geq 0, \\
x_{2}(t)=e^{t}, t \geq 0
\end{array}\right.
$$

They are obviously unstable.

Adding a control with the delay term in (76), we have control systems

$$
\begin{cases}\dot{x}_{1}(t)=x_{2}(t), & t \geq 0, \\ \dot{x}_{2}(t)=-x_{1}(t)+2 x_{2}(t)+u(t-h), & t \geq 0, \\ x_{1}(0)=1, & \\ x_{2}(0)=1, & -h \leq t \leq 0, \\ u(t)=\varphi(t), & \end{cases}
$$

where $x_{1}(t), x_{2}(t) \in R, u(t) \in R$, and $\varphi(t) \in R$ are the initial control function.

Comparing (78) with (1), we have

$$
\begin{aligned}
& A=\left(\begin{array}{cc}
0 & 1 \\
-1 & 2
\end{array}\right), \\
& B=\left(\begin{array}{l}
0 \\
1
\end{array}\right) .
\end{aligned}
$$

For

$$
\operatorname{Rank}[B, A B]=\operatorname{Rank}\left(\begin{array}{ll}
0 & 1 \\
1 & 2
\end{array}\right)=2,
$$

from Lemma 1, we have that system (78) is controllable.
For

$$
p(s ; A)=\left|s\left(\begin{array}{ll}
1 & 0 \\
0 & 1
\end{array}\right)-\left(\begin{array}{cc}
0 & 1 \\
-1 & 2
\end{array}\right)\right|=\left|\left(\begin{array}{cc}
s & -1 \\
1 & s-2
\end{array}\right)\right|=(s-1)^{2},
$$

use Theorem 2, for $a=1$ and $b=0$, we have that system (78) is stabilizable with delay $h \in[0, \pi / 3(2+\sqrt{3}))$.

We get an interval $[0, \pi / 3(2+\sqrt{3}))$ of the control delay, in which the stabilizability of system (78) can be kept.

We must take notice that if the control delay is outside of interval $[0, \pi / 3(2+\sqrt{3}))$, system (78) is perhaps unstable. In fact, if we take the feedback matrix as $K=(0,4)$, the control delay $h=6 \ln 2$ and system (78) is unstable. For $h=6 \ln 2=\ln 32>\ln e=1>(2 / 3)>4 / 3(1) /(2+\sqrt{3})>\pi / 3$ $\times(2+\sqrt{3}), h=6 \ln 2$ is outside of interval $[0, \pi / 3(2+\sqrt{3}))$.

For

$$
\begin{aligned}
p(s ; A, B K ; h) \triangleq \operatorname{det}\left(s I-A-B K e^{-h s}\right) \\
\quad=\left|s\left(\begin{array}{ll}
1 & 0 \\
0 & 1
\end{array}\right)-\left(\begin{array}{cc}
0 & 1 \\
-1 & 2
\end{array}\right)-\left(\begin{array}{ll}
0 & 0 \\
0 & 4
\end{array}\right) e^{-h s}\right| \\
\quad=\left|\left(\begin{array}{cc}
s & -1 \\
1 & s-2-4 e^{-h s}
\end{array}\right)\right|=(s-1)^{2}-4 s e^{-h s},
\end{aligned}
$$

$s=1 / 2$ satisfies equation

$$
p(s ; A, B K ; 6 \ln 2)=0 .
$$

System (78) has solutions

$$
\begin{cases}x_{1}(t)=e^{1 / 2 t}, & t \geq 0, \\ x_{2}(t)=e^{1 / 2 t}, & t \geq 0 .\end{cases}
$$

They are obviously unstable.

\section{Concluding Remarks}

In practice, we often meet many unstable systems such as

$$
\left\{\begin{array}{l}
\dot{x}(t)=A x(t), \\
x\left(t_{0}\right)=x_{0}, \\
t \geq t_{0}
\end{array}\right.
$$

where $x(t) \in R^{n}$ is a state vector. We usually add control terms in them. But unfortunately, due to the transmission of the signal or the mechanical transmission takes a period of time, time delay may appear in control variables, and the control systems will become as system (1). The results of this paper give the methods to find the interval of the control delay, in which the stabilizability of the systems can be kept. From Example 1, we can see that, on the outside of this interval, the systems may be unstable. So, the method given in this paper has practical significance.

Now, we give some Remarks to finish this paper.

Remark 1. By Lemmas 1-4 and Theorem 1, system (1) is controllable and 


$$
p(s ; A)=s^{n}+a_{n-1} s^{n-1}+\cdots+a_{1} s+a_{0}=0 .
$$

There exists some negative real part roots, and we can also get analogous results and find the interval of control delay with which the systems are stabilizable.

Remark 2. Although Theorem 4 requires system (1) to be controllable, if it is uncontrollable but the uncontrollable subsystems are stable, we can also get analogous results and find the interval of control delay with which the systems are stabilizable.

Remark 3. In Theorem 4 , interval $\left[0, \pi / 3 y^{*}\right)$ of control delay $h$ is obtained, with which the systems are stabilizable. But whether $\left[0, \pi / 3 y^{*}\right)$ includes all delay values for which system (1) is stabilizable can be left as an open problem.

Remark 4. In papers [5, 6, 15-20], the authors have given some excellent results for the stability of delay systems. But all systems, they study, are not control systems, we cannot use their results directly to judge the stabilizability for the control systems with control delay. In comparison with these, we can see that the results of this paper are very useful.

Remark 5. We note that, in recent years, many scholars researched delayed systems with fast time-varying delay $[20,21]$ and gave many wonderful consequences. Usually the methods they used to deal with stability are linear matrix inequalities (LMIs). In this paper, we use the characteristic root methods to deal with stability (or the stabilizability) for the systems with time-invariant delay. If we want to use the characteristic root methods to deal with the stability (or the stabilizability) for the delayed systems with fast time-varying delay, or to use the results of this paper to the delayed systems with fast time-varying delay, we will meet more difficulties.

\section{Data Availability}

No data were used to support this study.

\section{Conflicts of Interest}

The authors declare that they have no conflicts of interest.

\section{Acknowledgments}

This research was supported by the National Natural Science Foundation of China (nos. 11371027, 11471015, and 1601003), Anhui Provincial Natural Science Foundation (nos. 1608085MA12 and 1508085MA01), and Program of Natural Science of Colleges of Anhui Province (no. KJ2013A032).

\section{References}

[1] J. K. Hale, M. Sjoerd, and V. Lunel, Introduction to Functional Differential Equations, Springer, Berlin, Germany, 1992.

[2] W. Jiang, The Degenerate Differential Systems with Delay, Anhui University Press, Hefei, China, 1998.
[3] J. Wei, "Eigenvalue and stability of singular differential delay systems," Journal of Mathematical Analysis and Applications, vol. 297, no. 1, pp. 305-316, 2004.

[4] J. Wei and S. Wenzhong, "Controllability of singular systems with control delay," Automatica, vol. 37, no. 11, pp. 1873$1877,2001$.

[5] J. Chiasson, "A Method for computing the Interval of delay Values for which a differential-delay system is stable," IEEE Transactions on Automatic Control, vol. 33, no. 12, pp. 1176-1178, 1988.

[6] J. Jie Chen, "On computing the maximal delay intervals for stability of linear delay systems," IEEE Transactions on Automatic Control, vol. 40, no. 6, pp. 1087-1093, 1995.

[7] J.-P. Richard, "Time-delay systems: an overview of some recent advances and open problems," Automatica, vol. 39, no. 10 , pp. 1667-1694, 2003.

[8] L. Mirkin, "On the approximation of distributed-delay control laws," Systems \& Control Letters, vol. 51, no. 5, pp. 331-342, 2004.

[9] W. Jiang, "Function-controllability of nonlinear singular delay differential control systems," Acta Mathematica Sinica, vol. 49, no. 5, pp. 1153-1162, 2006.

[10] J. Wei, "The controllability of fractional control systems with control delay," Computers \& Mathematics with Applications, vol. 64, no. 10, pp. 3153-3159, 2012.

[11] H. Zhang, J. Cao, and W. Jiang, "Controllability criteria for linear fractional differential systems with state delay and impulses," Journal of Applied Mathematics, vol. 2013, Article ID 146010, 9 pages, 2013.

[12] X.-F. Zhou, F. Yang, and W. Jiang, "Analytic study on linear neutral fractional differential equations," Applied Mathematics and Computation, vol. 257, no. 2, pp. 295-307, 2015.

[13] N. Li and W. Jiang, "Convergence analysis of fractional iterative learning control nonlinear systems with multiple state delays," Pure and Applied Mathematics, vol. 33, no. 1, pp. 69-81, 2017.

[14] A. U. K. Niazi, W. Jiang, M. U. Rehman, and D. Pang, "Boundary value problem for nonlinear fractional differential equations with delay," Advances in Difference Equations, vol. 2017, no. 1, p. 24, 2017.

[15] J. Wei, "On the interval controllability of fractional systems with control delay," Journal of Mathematics Research, vol. 9, no. 5, pp. 87-96, 2017.

[16] A. U. K. Niazi, J. Wei, M. U. Rehman, and P. Denghao, "Ulam-Hyers-Mittag-Leffler stability for nonlinear fractional neutral differential equations," Sbornik: Mathematics, vol. 209, no. 9, pp. 1337-1350, 2018.

[17] J.-A. López-Renteria, B. Aguirre-Hernández, and F. Verduzco, "The boundary crossing theorem and the maximal stability interval," Mathematical Problems in Engineering, vol. 2011, Article ID 123403, 13 pages, 2011.

[18] B. L. Hernndez-Galvn, J. A. Lpez-Rentera, B. AguirreHernndez, and G. Fernndez-Anaya, "Robust stability in discrete control systems via linear, controllers with single and delayed time," Mathematical Problems in Engineering, vol. 2018, Article ID 3674628, 11 pages, 2018.

[19] J. A. Lopez-Renteria, B. Aguirre-Hernandez, and G. Fernandez-Anaya, "LMI stability test for fractional order initialized control systems," Applied and Computational Mathematics, vol. 18, no. 1, pp. 50-56, 2019.

[20] P.-L. Liu, "Delay-range-dependent stability criteria for takagisugeno fuzzy systems with fast time-varying delays," Journal of Applied Mathematics, vol. 2012, Article ID 475728, 20 pages, 2012. 
[21] X.-Y. Xi, J.-F. Zhao, T.-Z. Liu, and L.-M. Yan, "Fault diagnosis and fault-tolerant control for system with fast time-varying delay," Automatika, vol. 60, no. 4, pp. 462-479, 2019.

[22] W. Jiang, W.Z. Song, S. Fei, and S. Song, "On the controllability and stabilizability of control delay systems," in Proceedings of the 3rd World Congress on Intelligent Control and Automation, pp. 2846-2849, Press of University of Science and Technology of China, Hefei, China, 2000. 